\title{
The State of Information and Communication Technology and Health Informatics in Ghana
}

\author{
Emmanuel Kusi Achampong ${ }^{1}$ \\ ${ }^{1}$ University of Cape Coast, Ghana
}

\begin{abstract}
Information and Communication Technology (ICT) has become a major tool in delivery of health services and has had an innovative impact on quality of life. ICT is affecting the way healthcare is delivered to clients. In this paper, we discuss the state of ICT and health informatics in Ghana. We also discuss the state of various relevant infrastructures for the successful implementation of ehealth projects. We analyse the past and present state of health informatics in Ghana, in comparison to other African countries. We also review the challenges facing successful implementation of health informatics projects in Ghana and suggest possible solutions.
\end{abstract}

Keywords: Information and Communication Technology, health Informatics.

\section{Introduction}

ICT presents many opportunities for improving the performance of health systems in developing countries. This has been demonstrated in several pilot projects across the developing world ${ }^{1}$. The availability of affordable equipment has also contributed to the several initiatives aimed at improving the effectiveness of health care providers, the efficiency of health care managers and new opportunities for health care clients.

ICT has assisted in driving down healthcare costs ${ }^{2}$; and improved the delivery and effectiveness of healthcare services through help in disease management, improved patient safety and decision support for practitioners ${ }^{3}$. Various systems have been developed to aid health care delivery such as local area network based patient information systems ${ }^{4}$, and online health information for patients and medical personnel.

\section{State of ICT in Ghana}

We live in a world in which all aspects of life are influenced by ICT. In this paper, we discuss ICT as the driving force of health informatics. ICT is a tool that can aid dissemination of information through electronic media. We define ICT as a tool or technology for gathering, storing, retrieving, processing, analysing and disseminating information electronically. ICT is a fusion of telecommunication and computing with the aim of processing and disseminating information. There are many factors that determine the implementation and use of ICT such as ICT expert knowledge, user's attitudes, etc. 
Since the first internet connection was set up in 1989, Ghana has been a regional leader in ICT $^{1}$. Over the past two decades the government and the private sector have worked together to maintain this lead. The state's on-going ICT for Accelerated Development (ICT4AD) programme has resulted in numerous improvements in the sector and the economy as a whole. The plan which is now being redesigned to meet the current needs of the rapidly changing ICT sector is expected to play a major role in the coming years.

The private ICT sector is highly competitive. Ghana is home to about 180 internet service providers (ISPs), though business is concentrated among a handful of large firms, and only around 30 companies are currently active. Internet penetration estimates range from $4 \%$ to $6 \%$, but most major local players and multinational ICT monitoring organisations concur that the nation has one of the highest ICT penetration rates in sub-Saharan Africa. Most people access the internet at the country's numerous internet cafes, though thanks to rising incomes throughout the country a steady increasing number of Ghanaians are able to afford an internet connection at home ${ }^{1}$.

The nation serves as a landing point for a number of huge submarine communications cable projects such as GLO1, SAT3, and WASC. These will boost overall connectivity and network speeds and push down prices. Additionally, both the government and private sector players are working to increase internet awareness among the wider population. With these efforts in mind, internet penetration is expected to increase dramatically over the next decade 1

\section{Government Influence in ICT}

A number of state organisations are involved in ICT in Ghana. The Ministry of Communications $(\mathrm{MoC})$ oversees general policy and state-sponsored initiatives. The National Information Technology Agency (NITA), established in 2008, is the MoC's ICT development and implementation arm. As of early 2011 NITA was working to revitalise ICT4AD to better reflect the country's current needs ${ }^{1}$.

The ICT sector is regulated by the National Communications Authority (NCA), which was set up in 1997 as the result of new telecommunications legislation passed the previous year. Ghana's reputation as one of the most liberal ICT markets in Africa is largely the result of pro-competition policies put in place by the NCA. In March 2011, the NCA introduced a bill that would give the authority new powers over ICT and telecoms companies, including the right to approve acquisitions and other large share purchases. The bill is currently under discussion in parliament. In addition, NCA is also working to advance the spread of highspeed internet services throughout Ghana.

\section{Ownership of Computers}

Personal computers (PC) and laptops are largely the main equipment used to exploit the potential of the internet and networked services. Therefore, it is important to determine the level of ownership of computers. From a survey, only 5.0\% of the sample from Ghana had a PC/laptop at home ${ }^{5}$ and this formed one-third of the situation in South Africa. Though there has been slight improvement in the ownership of PC from an earlier survey which showed that only $4 \%$ of the sample had computer/laptops at home ${ }^{6}$, the fact still remains that ownership of the technology is very poor in the country. In spite of the global fall in prices and a seeming growing trade in used computers in the country, these have not significantly 
improved ownership of computers in the country. Becoming computer literate is one of the basic prerequisites of participating in the digital revolution as well as achieving the objective of the national ICT4AD Policy.

Regretfully, most household computers are not connected to the internet. A survey showed that only $0.3 \%$ of the households in Ghana had internet connection at home ${ }^{5}$. This is far below countries such as South Africa (4.8\%), Namibia (3.3\%), Kenya (2.2\%) and Cameroon $(1.18 \%)$. This has serious implication in widespread use of the service at the household level. Internet at home will help students, as well as parents to utilise the vast potential of the service and its lack at residential level is a major limiting factor to exploiting networked services 5 .

Notwithstanding, computers have been available at various workplaces in hospitals and health centres. The challenge has been with the skill to use these computers for its intended purpose. Secondly, connectivity at the various health centres has been a major challenge.

\section{ICT Infrastructure}

Computing equipment (desktops, laptops, servers), networking devices (switches, routers, wireless access points, firewalls, local area networks (LANs) and wide area networks (WANs)), multimedia systems (Television sets, VCD and DVD players, camera's and camcorders), mobile telephony and communication (Personal Digital Assistants (PDAs), cell phone, landlines, fax machines etc.), imaging (desktop and network printers) and internet systems (GPRS, ADSL, VSAT and modems) form the basis for ICT infrastructure ${ }^{1}$.

Mostly every health facility has computing equipment, multimedia device, imaging and printing system, communication and internet system. The existing ICT infrastructure have not been fully integrated and networked in a manner to support the healthcare delivery system ${ }^{1}$.

Apart from few hospitals with a fully functional local area network (LAN), most of the healthcare providers have restricted their LANs to the front office, records and pharmacy departments/units of their facilities. The LANs are mainly used to support the automation of pharmacy department/units and front office operations like patient registration and records keeping ${ }^{1}$.

Multimedia systems are employed for playing back medical and non-medical documentaries and movies at the front desks.

Routine planned preventative maintenance is not carried out because of budgetary constraints. Service level agreement is not used to define the nature and quality of ICT services outsourced.

\section{ICT Policies in Ghana}

There are a number of ICT policies that have been put in place to facilitate the quick implementation of ICT projects in the country.

There is the Information and Communication Technology for Accelerated Development (ICT4AD) Policy which is geared towards improving the socio-economic status of the 
citizens including healthcare. The ICT4AD policy represents the vision for Ghana in the information age.

There is the Health Sector ICT policy and ehealth policy for the country. The goal of the ehealth strategy is to harness the potential of ICT to improve the health status of people living in Ghana.

\section{Telephony System in Ghana}

The telecommunication sector of Ghana is one of the most liberalised markets in Africa. The market has two national fixed-network operators and six operating mobile telephone companies. The fixed-line telephone segment is almost a monopolistic market since Vodafone Ghana controls almost $98 \%$ of the market, while Airtel, the second network provider, has only $2 \%$ market share.

Unlike the fixed-line telephone market, there is rigorous competition in the mobile telephone market and this has contributed to an improved penetration rate in the country. In 2008, the telephone penetration stood at $52.4 \%$, of which mobile telephones contributed $99 \%$. The analysis showed that the deployment of fixed-line telephones has been on the decline and between 2003 and 2008; it experienced a negative Compound Annual Growth Rate (CAGR) of 13.1 , while mobile telephones had a positive CAGR of $70.8 \%{ }^{7}$.

\section{Internet in Ghana}

In August 1995, Ghana became the second country in sub-Saharan Africa to have full internet connectivity. The country is directly connected to the world's first submarine fibre-optic cable system, SAT-3/WASC/SAFE, which links Africa to Europe and Asia ${ }^{12}$. Network Computer Systems Ltd (NCS) established the first connection. NCS was given its own VSAT (very small aperture terminal) gateway as a result of the constraints experienced by the then Ghana Telecom (GT). By 2002, the National Communication Authority (NCA) had licensed 52 internet service providers (ISPs), although only about ten were operational at that time ${ }^{8}$.

There have been several e-mail systems in Ghana over the years, which have been based primarily on FidoNet and Unix to Unix CoPy (UUCP). UUCP refers to a suite of computer programs and protocols allowing remote execution of commands and the transfer of data files and e-mail between computers. Ghana gained access to FidoNet, an early international bulletin board system, in 1989, under the aegis of a programme sponsored by the UN's Economic Commission for Africa and the Canada-based International Development Research Centre. In the early 1990's the system was upgraded, and access was expanded to include 23 scientific, governmental and educational entities. By 1995 the ".gh" domain name had been registered with the Internet Assigned Numbers Authority (IANA), the international organisation that coordinates top-level internet protocol resources. Much of this development was undertaken by the then Ghana Telecom (GT), which was launched by the government in1974 and served as the national telecoms operator until 2008, when $70 \%$ of the firm was sold to Vodafone ${ }^{1}$.

As of mid-2009 there were around 47,000 internet subscribers in Ghana, which makes for a penetration rate of just $0.2 \%$, according to Internet Research, a local ICT research firm. Most internet subscriptions are held by businesses or internet cafes, and so are used by multiple individuals. Consequently, the total number of internet users in Ghana is actually much 
higher, though estimates vary widely. According to the International Telecommunication Union (ITU), the UN's ICT development agency, as of June 2010 Ghana had $1.3 \mathrm{~m}$ internet users, which translates as a penetration rate of 5.3\%, up from 997,000 (4.2\%) in 2009, $880,000(3.8 \%)$ in 2008, 401,300 (1.8\%) in 2006 and just 30,000 (0,2\%) in 2000. The 2010 penetration rate is among the highest in the sub-Saharan region, but lower than the African average of $10.9 \%$, though this is skewed by the North African countries, the majority of which have internet penetration of $20-30 \%{ }^{1}$.

In 2009, 58\% - 27,399 subscribers - of all internet subscriptions were for high speed broadband packages, an overall penetration rate of around $0.1 \%$, according to Internet Research, a local ICT firm. By 2015, National Broadband Strategy, which falls under the aegis of ICT4AD, seeks to increase national broadband penetration to $50 \%$, reduce the cost of broadband by $80 \%$ and lower the cost of broadband-related hardware, including PCs, by $90 \%$. In addition to improving high-speed internet access and the programme is also expected to boost GDP growth ${ }^{1}$.

Outside Ghana's urban centres, internet penetration falls off dramatically. Most of Ghana's rural inhabitants access the web through their mobile phones or not at all. Estimates for internet penetration in rural Ghana hover around $1 \%{ }^{1}$.

The internet has become a very useful infrastructure in utilising the opportunities of the digital revolution. Technological convergence and the development of multi-media services and increasing business applications of the internet have made access and usage a fundamental issue in the participation in the information society.

\section{Ghana Health Informatics: Past and Present}

Health informatics in Ghana is at the infant stages. A lot of informatics projects are disjointed and there is no coordination between these projects. There are whole lots of pilot projects that are running in the country. Pilot projects are being implemented in some hospitals with respect to Health Management Information System (HMIS). Most hospitals in the country are partially electronic. Efforts to establish an adequate management information system throughout the health sector have been on-going for some time now.

Examining the current state within the Health Sector, it can be concluded that the essential building blocks for establishing a proper HMIS are in place:

- HMIS Strategic Plan

- Policy and Legal framework for health data reporting

- Medical records policy

- Framework for a central data repository

- Computerised District Health Management Information System

- The establishment of a Centre for Health Information at central level

\section{Health Management Information Systems}

The use of health management information system is not widespread. There are currently two main applications used for information management. One version is used for the management of clinical business process whilst the other supports the collection and aggregation of data, 
and is mainly used for reporting. PDAs are sometimes used for collecting data at the district and community levels.

The District Health Management Information System (DHIMS) software application was primarily used for data capture, aggregation and generation of management reports. It has been replaced with the District Health Information System (DHIS2) which is being deployed for data capture, aggregation and generation of management report.

Different software is being used by various hospitals. These software applications are modular in nature and do not support the full processes in the hospitals.

The extent to which these processes are supported by information systems varies from hospital to hospital and from activity to activity. The characteristics of the hospital processes are as follows:

- Documentation on admission, capturing of treatment regimen and discharge summaries are all done manually and kept in folders

- Key elements of the patient record in hospitals remain exclusively paper-based.

- Imaging and diagnostic results are not available electronically and are also not accessible from remote locations.

- Electronic versions of prescription history is not available

- There is no electronic logistics and supply chain management system in place for medicines and non-consumables. Most activities are carried out manually.

- There are no systems for the generation of electronic medical records. This impact significantly on the arrangements for referrals.

- Long waiting time for patients

- Inadequate staffing levels - clinical and non-clinical

- Challenges with financial management reporting

\section{National Health Insurance Scheme (NHIS)}

The Government's health insurance scheme plays a vital role in the financing arrangements for the health care providers. The National Health Insurance Authority (NHIA) has deployed an ICT infrastructure for the automation of health insurance services. All accredited healthcare providers operate a common ICT platform with common protocols for patient's authentication and claims management. There are also plans to deploy an online claims management system.

The ICT platform for the national health insurance does not currently support any shared services. It meets only the business processes and needs of the NHIS.

\section{Human Resource System}

The availability of staff with clinical and non-clinical background and a desirable level of IT literacy are crucial in any health informatics project. The Ministry of Health $(\mathrm{MOH})$ does not have adequate staffs with skill sets in health informatics systems. The few staff who have undergone professional IT training are not involved in mainstream ICT related activities because the existing Human Resource establishment post does not have a structure for ICT professionals. 
There is no routine or formal training for professionals with clinical and non-clinical background in ICT related courses like networking, systems administration, database administration, security, fundamental of computing and web-based systems, etc.

\section{Diagnostic Information systems}

Several health facilities have diagnostics and imaging equipment. This equipment is used for the conventional laboratory examinations and X-rays. The use of paper-based documentation for the diagnostics and films for x-rays are the standard practice. Technicians usually carry out tests whilst physicians or other specialist handle the interpretations of results.

Even though some diagnostic and imaging equipment support automation and have the capacity for digital imaging they are hardly used for that purpose.

\section{Telemedicine and eLearning}

Telemedicine is the use of information and communications technology to deliver healthcare services, promote healthy lifestyles and education, where the patients or clients are geographically separated. Telemedicine facilitates clinical consultation including patient assessment, diagnosis and treatment, continuing professional education, health promotion, and healthcare management.

There is currently a national coverage for telecommunication via landlines and cell phone. Internet connectivity is accessible nationally. It is available via ADSL, GPRS and VSAT. All the ISPs have internet enable features on their network. The Ministry of Communication is rolling out a programme for optical fibre connectivity for all regional hospital and selected district hospitals.

A national wide area network (WAN) is also being deployed by the Ministry of Communication for use by the Municipal and District Assemblies (MDAs). There are however challenges with bandwidth and cost.

There is clear commitment from the top management to establish a robust and dependable telecommunication infrastructure throughout the country. In spite of the on-going activities, there is still a large "digital divide" delineated by geography, income, education level, literacy, etc.

eLearning is practiced on a very small scaled. Even though eLearning is recognised by the $\mathrm{MOH}$ as one of the means of carrying out cost effective, efficient and convenient medical education, the medical schools and training centres have been slow in changing their teaching methodologies. No training institution has an eLearning-enabled training curricular with interactive features and online tutor support functionalities.

The only eLearning project is the Pan African eNetwork Project at the Kwame Nkrumah University of Science and Technology (KNUST). The University as an academic institution and the University's hospital are being used as the project site. Participation and connectivity to the eLearning project is restricted to a few selected sites in Africa and India. Other local hospitals and universities are not allowed to connect to the network.

\section{Security, Data Protection and Confidentiality}


There is currently no policy guideline with respect to electronic data interchanges and patient identifiable information in the health sector. Ownership of electronic data and information is not defined.

\section{Mobile Technology for Community Health (MoTeCH)}

MoTeCH explores the potential for health service benefits of information technology. The goals of the project include:

- Reviewing existing health information systems and assessing community health service information needs

- Developing a community service delivery system for testing mobile phone-based information systems for improving maternal, new-born, and early childhood care;

- Developing the district health management system software that is needed to accommodate mobile phone-transmitted service information;

- Evaluating the impact of mobile phone technology introduction on health service volume and delivery;

$\mathrm{MoTeCH}$ utilises a versatile platform that is not specific to maternal or child health care needs. MoTeCH uses OpenMRS, which is an open source medical record system as the basis of the medical records management. It has added "rules engine" and other components to process both inbound and outbound text and voice messages 9 .

The MoTeCH program provides expecting and recent mothers with valuable resources including:

- The Mobile Midwife Initiative: Mobile Midwife is an innovative program which provides expecting or new mothers accurate health education and reminders of upcoming clinic check-ups for themselves or their babies,

- The option to use either SMS or voice facility

- Several language choices including English, Kassim, Nankam (local languages at the pilot project's locations)

- Alerts or notifications about upcoming appointments at the clinic. This in turn reduces the number of defaulters (patients overdue for check-ups)

- MoTeCH enables nurses to directly enter patient encounter information into their mobile phones. This information is then used in the generation of the nurses' monthly reports. This automatic generation of reports saves the nurses' valuable time and improves report accuracy.

- The MoTeCH system's search component further allows nurses to search for patients' records through their mobile phone. On an individual level, a nurse can look for specific information on a patient, such as their due date or contact information. The system also allows for nurses to search information about groups of patients in their catchment area. An example of this would be a search for all defaulters (patients overdue for check-ups) or a listing of women with upcoming due dates.

- Another valuable tool that MoTeCH provides nurses with is the alert or automated SMS functions. On a weekly basis nurses are notified via text messages on various patient updates such as new defaulters and both upcoming and recent deliveries. This can allow for nurses to work together more efficiently in planning ahead for upcoming deliveries of care ${ }^{9}$. 


\section{SENE Pocket Digital Assistant (PDA) PROJECT- AN e-health INITIATIVE IN GHANA}

The use of mobile devices in the health sector in Ghana has been increasing over the past five years. The Sene PDA Project is one of the pioneer mobile health projects in Ghana and indeed the very first mobile project within the Ghana Health Service.

The Sene District PDA Project involves the use of Pocket Digital Assistants (PDAs) to collect public health service data at the lowest level of service delivery in Ghana Community-based Health Planning and Services (CHPS) zones. It is in the form of medical records to aid in following up clients to ensure continuity of care. The project also aims to produce accurate service data and reduce the time spent by service providers to compile inaccurate monthly data. The project has reached an advance stage in collecting data and use of data collected to ensure that every registered child completes his/her immunisation. The safe motherhood aspect of data collection i.e. antenatal care supervised delivery and postnatal care has been started but it is still in the rudimentary phase ${ }^{9}$.

Data is collected by the Community Health Officers at the CHPS zones by registering each child who receives immunisation service. The demographic details of the children are taken using the PDA. The children are given unique identification numbers generated by the community health nurse. Address of the child is captured so that the child can be traced for home visits.

Every month this register is used to follow up children who are due for immunisation in the communities. For each community visit that the community health officer makes for an outreach clinic, she queries the database and has the names of the children who are due to be vaccinated and the type of vaccines they will be taking. This helps in the preparation in getting adequate vaccines for an outreach and also ensuring that she identifies and vaccinate every child in the community who is due to be vaccinated. This same data is synchronised with a computer at the District Health Directorate and an interface is used to generate the monthly immunisation facility report as required by the program. The generation of this electronic monthly immunisation reports has been estimated to save the community health nurse about five working days every month of report preparation, which can be used for service delivery. District Health Management Teams also gets immunisation reports promptly in order to make decisions on which community health nurses need extra support to reach their target populations ${ }^{9}$.

\section{Health Informatics: Comparing Ghana and Nigerian Experiences}

In the context of developing nations, the use of ICT can potentially improve delivery of health care, patient care and reduce cost of running hospital ${ }^{10}$.

Ghana is still behind because of infrastructural issues that confronts the nation in respect to telecommunications. Other challenges include unstable power supply, inadequate telecommunication facilities, unskilled staff and many others. In Ghana, all regional hospitals have some form of computer networking within their facility with the intent of creating $100 \%$ connectivity. 
Most major hospitals in Ghana have either computerised their front desks, records or pharmacy departments. There are many partially computerised systems in the country where one of many departments is computerised to make the department's work easy. Although various hospitals have computerised record sections, paper folders are still used by patients. Very few hospitals in the country have fully functional electronic health records system. The National Health Insurance Scheme in Ghana which was introduced in 2006 has introduced computer processing of claims into their processes. Computers have been secured for this online claim processing at the various major hospitals.

In 2005, the Nigerian government also embarked on a similar system which is called the National Health Insurance Scheme (NHIS). Arguably, establishment of the scheme ought to have begun with the introduction of ICT in hospitals because ICT will reduce the cost of running the hospital and improve the delivery of healthcare system if fully implemented ${ }^{3}$.

In Nigeria, most hospitals have no electronic patient records; files are kept at the medical records section of the hospital. Sometimes, some of the files go missing within the hospital, making it difficult for physicians to trace the medical history of patients. Although few researchers ${ }^{11}$ have developed systems to solve referral system in the hospitals but the system were yet to be implemented because the Nigerian government has yet to show interest in any ICT investment in any hospital.

In Nigeria, the physicians in the same hospital cannot share a patient's file within the hospital and an electronic booking service is not available in any hospital in Nigeria. Sharing of patient's file is very difficult in most Ghanaian hospitals which still use paper records. There are no medical libraries to refer to by the physician online since most of our hospitals are not connected to the internet. This type of facility is not available in Nigeria. Evidence-based medicine which is being championed in the west has still not being implemented since health care facilities are not ready for such purpose.

There are no computer-based appointments systems in both Ghana and Nigeria; patients are always expected to visit the hospital to see the doctor without any prior communication with the doctor. The availability of internet has made it easy and fast to access health information, health advice and care through online information systems aimed at the public. Ghana has over $95 \%$ penetration rate of mobile telephony and this is being exploited to provide quality health care to patients. All the teaching hospitals in Ghana have some computer network facility that is being used for internal communication. Nowadays, hospitals could potentially use mobile phone technology to offer services to patients, but maintenance of the service requires funding.

\section{Challenges to Health Informatics in Ghana}

In Ghana, there are four main challenges to the use of ICT and the successful implementation of health informatics projects. These challenges stem from three factors, namely people, government and ICT infrastructure. These challenges are discussed as follows.

\section{i. Electric Power Supply}

Ghana has a moderately stable power supply as compared with other neighbouring countries. Any country that finds it difficult to provide Uninterrupted Power Supply (UPS) to its citizens will definitely have problems with deployment of good ICT services. In Ghana, one cannot fully rely on the electric power being supplied by the Electricity Company of Ghana since at any time without notice power can go off. ICT equipment was made to function with 
other infrastructure such as electricity under "controlled conditions" that is when electricity supply is stable and constant.

ii. Internet connectivity

The Internet help in controlling cost and more importantly it transform the flow of information in health sector. Healthcare organisations use internet for business processes due to cost reduction which was estimated at 10:1 to $100: 1$ in routine transactions ${ }^{3}$. There is a national fibre optic project that is being implemented by the government which will connect all regional and district hospitals. This is being done as part of the e-governance project which is being implemented by the Government of Ghana.

iii. Resistance to New Technology

The introduction of new technology is related to the user of the technology which may be positive and negative. Many Ghanaians, like any other citizens of the world, will resist new technological developments which may threaten their job. The ideal is for new technology to be associated with new knowledge and skills, through training on how to use the technology. Workers expect training on how to use new technology and a corresponding increase in their income, while the organisation introducing new technology expectation may be to reduce staff strength and cost of operation. Often in Ghana, downsizing is the issue to be raised before the introduction of new technology and this always leads to resistance by the workers, because of the fear of losing their job. In order to use ICT in Ghanaian hospitals, government should train hospital staff on how to use it and not lay them off and employ those with ICT skills.

iv. Lack of Maintenance Culture

Lack of maintenance culture is another problem; even government agencies find it difficult to maintain ICT equipment in Ghana. Both preventive and corrective maintenance is very important for any ICT equipment. The financial plans for purchases of any equipment should encompass the maintenance of such equipment, and allowances for depreciation in value which is not the case in many organisations in Ghana. This could be enforced by an ICT policy banning any organisation from importing, supplying and installing any ICT equipment without maintenance agreement.

\section{Benefit of ICT in Health Sector}

ICT has benefited the health sector both in developed nations and developing nations, the benefits affect the hospital's stakeholders: management of the hospital, health personnel and patients.

The use of ICT in health sector reduces the cost of running hospitals ${ }^{2}$. ICT introduces the potential sharing of patients' files easily without any threat to patient privacy. It is used for hospital management such as admission and appointment management.

ICT can also improve the efficiency of medical personnel by reducing waiting times and minimising paperwork. ICT makes information available for the use of hospital personnel in an easily readable form. The result of patients' test can be added to the patients' case file as soon as they are ready. To the patients, ICT gives 24-hour access to health information and through encryption and password protection can help to keep patients' data confidential. 


\section{Conclusion}

The state of ICT in Ghana is comparable with other developing nations. Out of all the ICT indicators, mobile phone has the highest number of users in Ghana. In Ghana, mobile phone has the highest number of users ${ }^{7}$ followed by the computer ${ }^{5}$. In this paper, we have discussed the challenges facing the use and implementation of ICT in Ghana which also have direct effect on health informatics in Ghana. Since "health is wealth", there is a need for Government of Ghana to make use of ICT to improve the delivery of healthcare so as to reduce poverty. NITA should be tasked to oversee the implementation of all ICT projects in the various regional and district hospitals in the country.

\section{Corresponding Author}

Emmanuel Kusi Achampong

University of Cape Coast, Ghana

Email: eachampong@ucc.edu.gh

\section{References}

1. Ghana eHealth Policy. Ministry of Health, Ghana. (2010).

2. Remlex D. Information and Communication Technology in Chronic Disease care. Medical Care Research and Review. 64(2). (2007). P.123-147

3. O’Carroll, P. W.; Yasnoff, W. A., Ward M. E.; Ripp, L. H.; Martin, E. L.(eds.). Public Health Informatics and Information Systems, Springer. (2003).

4. Modai I., Ritsner M., Silver H., \& Kurs R. A Computerized Patient Information System, Psychiatric Service, American Psychiatric Association, Volume 53, (2000). p. 476-478

5. Frempong. G. Developing information society in Ghana. How far? Electronic Journal of information systems in developing countries. (2011).

6. Frempong, G.K., Esselaar, S., Stork, C. \& Anyimadu, A. Household and Individual ICT Access and Usage in Ghana, in Gillwald, A. (Ed.), African e-Index, AXIUS Publisher, South Africa. (2005).

7. ITU. Measuring the Information Society: The ICT Development Index. (2009a). http://www.itu.int/ITU-D/ict/publications/idi/2009/index.htm.

8. Lundkvist. P., Habchi, D., Soderberg, B., Jensen, M., Akrof, E.O. \& Spintrack, C.D.E.. Fostering and facilitating access on the SAT-3/WASC/SAFE fibreoptic cable in West Africa: Improving West African internet connectivity using fibre optic cable. Stockholm: Spintrack IT Advice. [Online]. (2004). Available: www.spintrack.com/itadvice.

9. Ghana Health Service Publications http://www.ghanahealthservice.org/includes/upload/publications/MOBILE\%20TECHNO LOGY\%20FOR\%20COMMUNITY\%20HEALTH.pdf

10. Mbananga, N., Madale, R. \& Becker P. Evaluation of Hospital Information System in the Northern Province in South Africa Using Outcome Measure, Report prepared for the Health Systems Trust, The Medical Research Council of Journal of Health Informatics in Developing Countries www.jhidc.org Vol.2 (2002). No.1 • Jan 08 Page 23 South Africa, Pretoria. 
11. Idowu, P.A., Adagunodo, E.R. Aderounmu G. A. and Ogunbodede E. O. Electronic Referral System for State Hospitals in Nigeria. Ife Journal of Science, Volume 6 (2), (2004). p.161-166.

12. Research-Africa.net (n.d.) Ghana science and technology system: A brief profile. [Online]. Available: www.research-africa.net/media/pdf/Ghana-ST.pdf

13. Frempong, G.K. \& Braimah, I. Assessing Universal Access to ICTs in Ghana, The Encyclopedia of Developing Regional Communities with ICT, Idea Group Inc., Pennsylvania. (2005). 